\title{
High-temperature superconductivity and its robustness against magnetic polarization in monolayer $\mathrm{FeSe}$ on $\mathrm{EuTiO}_{3}$
}

\author{
Chong Liu (iD) ${ }^{1,2,5 凶}$, Hyungki Shin (iD $^{1,2,5}$, Andrin Doll ${ }^{3}$, Hsiang-Hsi Kung ${ }^{1,2,4}$, Ryan P. Day (iD ${ }^{1,2}$, Bruce A. Davidson ${ }^{1,2}$, Jan Dreiser ${ }^{3}$, \\ Giorgio Levy ${ }^{1,2}$, Andrea Damascelli, ${ }^{1,2}$, Cinthia Piamonteze ${ }^{3}$ and Ke Zou (D) ${ }^{1,2}$
}

Spin degree of freedom generally plays an important role in unconventional superconductivity. In many of the iron-based compounds, superconductivity is found in close proximity to long-range antiferromagnetic order, whereas monolayer FeSe grown on $\mathrm{SrTiO}_{3}$, with enhanced superconductivity, exhibits no magnetic or nematic ordering. Here we grow monolayer and multilayer FeSe on antiferromagnetic $\mathrm{EuTiO}_{3}(001)$ layers, in an effort to introduce a spin polarization in proximity to the superconductivity of FeSe. By X-ray magnetic dichroism, we observe an antiferromagnet-ferromagnet switching on Eu and Ti sites in EuTiO ${ }_{3}$ driven by the applied magnetic field, with no concomitant spin polarization on the Fe site of FeSe. Transport measurements show enhanced superconductivity of monolayer $\mathrm{FeSe}$ on $\mathrm{EuTiO}_{3}$ with a transition temperature of $\sim 30 \mathrm{~K}$. The band structure revealed by photoemission spectroscopy is analogous to that of $\mathrm{FeSe} / \mathrm{SrTiO}_{3}$. Our work creates a platform for the interplay of spin and unconventional superconductivity in the two-dimensional limit.

npj Quantum Materials (2021)6:85; https://doi.org/10.1038/s41535-021-00388-5

Spintronics ${ }^{1}$ offers the potential for creating circuits in which logic operations controlled by spin currents are faster and more energetically efficient than equivalent charge-based semiconductor devices. It requires control of spin and charge at the nanoscale, allowing devices with a greater diversity of functionality. The idea of combining superconductivity with the spin degree of freedom started in 1966 with a concept of magnetic memory, in which the superconducting transition temperature $\left(T_{\mathrm{c}}\right)$ of a thin-film $\mathrm{s}$-wave superconductor sandwiched between ferromagnetic insulators is controlled by the magnetization alignment ${ }^{2}$. Subsequently, attention has been focused on devices designed around materials in which the coexistence of magnetism and superconductivity is an intrinsic property or is induced at the interface by proximity ${ }^{3,4}$.

Two-dimensional (2D) materials that are only a few atomiclayers thick serve as a potential platform to combine superconductivity and magnetism. To date, their coexistence in the $2 \mathrm{D}$ limit has been discovered in $\mathrm{LaAlO}_{3} / \mathrm{SrTiO}_{3}{ }^{5-8}$ and $\mathrm{LaAlO}_{3} / \mathrm{EuTiO}_{3} /$ $\mathrm{SrTiO}_{3}$ interfaces ${ }^{9}$. The buried interface and low $T_{\mathrm{c}}(<0.3 \mathrm{~K})$ have limited the study and potential control of the coupling between spin and charge. A recent study casts some doubt on the origin of the ferromagnetism of $\mathrm{LaAlO}_{3} / \mathrm{SrTiO}_{3}$, suggesting that magnetism is induced by extrinsic defects and not an intrinsic property of the interface ${ }^{10}$.

In this report, we study the epitaxial interface between a 2D high-temperature superconductor-monolayer $\mathrm{FeSe}$-and an antiferromagnetic (AFM) insulator- $\mathrm{EuTiO}_{3}$ (ETO), which allows direct access to study and control the properties through all the standard techniques suitable for 2D materials. The role of the spin degree of freedom in this high-temperature iron-based superconductor is carefully examined to understand its relation to superconductivity.

Undoped bulk FeSe is superconducting below $8 \mathrm{~K}^{11}$, above which only nematicity but no long-range magnetic order is present at ambient pressure ${ }^{12-14}$, although strong spin fluctuations have been found by inelastic neutron scattering ${ }^{15}$. Monolayer FeSe shows strongly enhanced superconductivity with $T_{c}$ up to $70 \mathrm{~K}$ when epitaxially grown on $\mathrm{SrTiO}_{3}$ (STO) ${ }^{16-20}$ and other oxides ${ }^{21-27}$. Several mechanisms have been related to this emerging superconducting phase ${ }^{28}$, including spin fluctuations ${ }^{29-31}$, electron doping $^{32-35}$, and electron-phonon coupling ${ }^{36-41}$. Theoretical calculations proposed that the ground state of monolayer FeSe/ STO is checkerboard antiferromagnetic order and that it further hosts a quantum spin Hall state ${ }^{42,43}$, although no such experimental observation has been reported so far. Magnetic exchangebias measurements found evidences of antiferromagnetic order in as-grown FeSe thin films on STO; however, upon annealing, the sample became superconducting and AFM order disappeared ${ }^{44}$. Resonant inelastic X-ray scattering revealed dispersionless and significantly hardened spin excitations in monolayer FeSe/STO ${ }^{45}$. How the magnetic texture competes with the superconductivity in this system remains an open question.

Pioneer works choosing different substrates for FeSe, such as $\mathrm{FeSe} / \mathrm{BaTiO}_{3}{ }^{27}$, demonstrate that interface engineering is a powerful approach to tuning the properties. Here, we introduce magnetic ordering adjacent to FeSe thin layers by the interface with $\mathrm{EuTiO}_{3}$ using molecular beam epitaxy (MBE). EuTiO 3 is known to be a G-type antiferromagnetic insulator with a large spin moment of $7 \mu_{\mathrm{B}}$ per $\mathrm{Eu}^{46}$ and Néel temperature $T_{\mathrm{N}}$ at $\sim 5.5 \mathrm{~K}^{46,47}$. It has a perovskite-type cubic structure with lattice constant $a=$ $3.905 \AA$, identical to STO, allowing high-quality growth of FeSe films. Combining X-ray absorption spectroscopy, electrical and magneto- transport, and angle-resolved photoemission spectroscopy (ARPES) measurements, we detected large tunable spin polarization in ETO and, simultaneously, robust high- $T_{\mathrm{c}}$ superconductivity in monolayer FeSe/ETO with no magnetic order detected on Fe sites.

\footnotetext{
${ }^{1}$ Quantum Matter Institute, University of British Columbia, Vancouver, British Columbia V6T 1Z4, Canada. ${ }^{2}$ Department of Physics and Astronomy, University of British Columbia, Vancouver, British Columbia V6T 1Z1, Canada. ${ }^{3}$ Swiss Light Source, Paul Scherrer Institut, CH-5232 Villigen PSI, Switzerland. ${ }^{4}$ Max Planck Institute for Chemical Physics of Solids, Nöthnitzer Straße 40, Dresden 01187, Germany. ${ }^{5}$ These authors contributed equally: Chong Liu, Hyungki Shin. ${ }^{凶}$ email: chong.liu@ubc.ca; kzou@phas.ubc.ca
} 

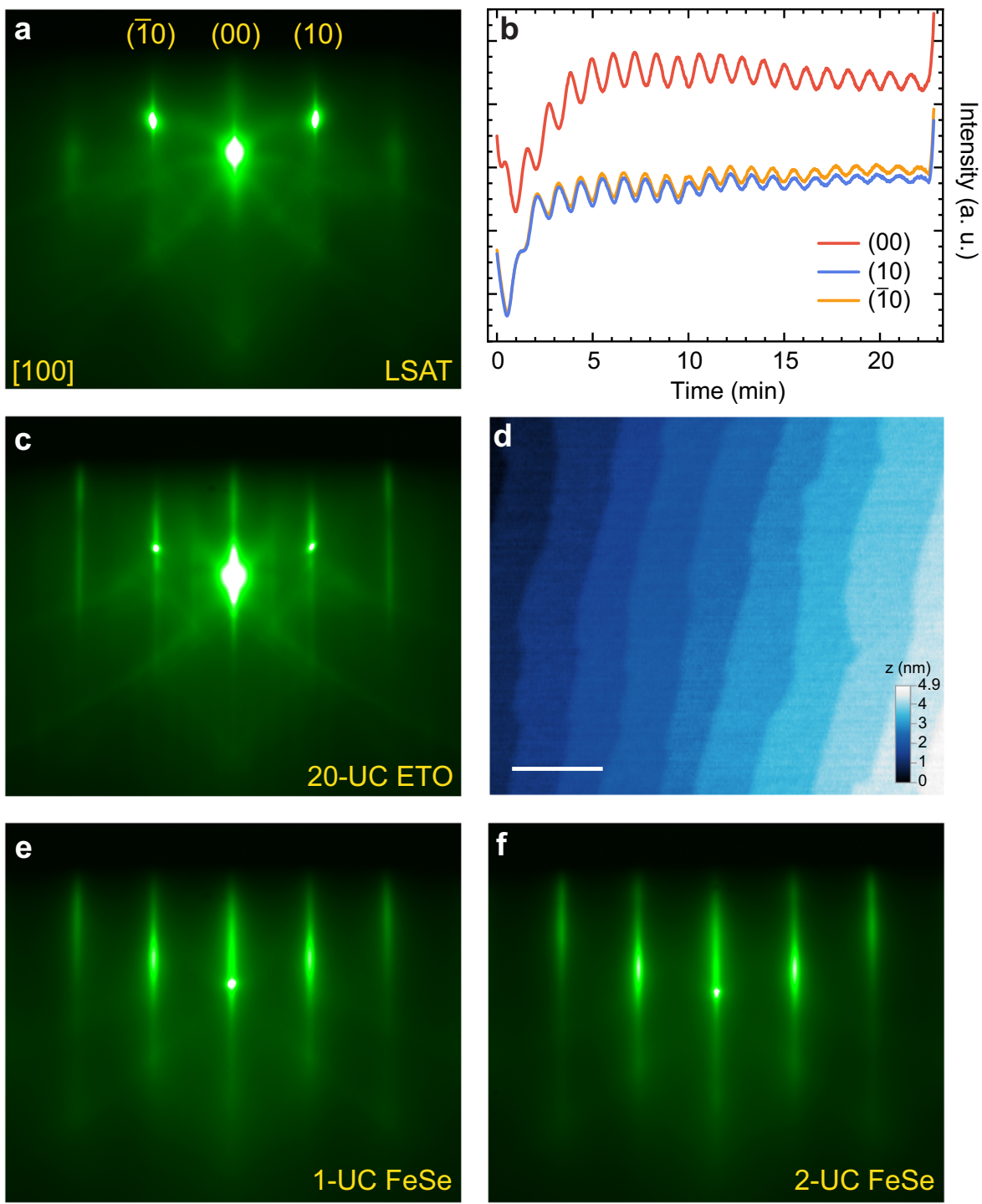

Fig. 1 MBE growth of the films. a RHEED pattern of the LSAT substrate. b RHEED oscillations during the growth of the 20-UC ETO. c RHEED pattern of the 20-UC ETO on LSAT. d Atomic force microscope image of the 20-UC ETO. The length of the scale bar corresponds to $200 \mathrm{~nm}$. e RHEED pattern of 1-UC FeSe on ETO. $\mathbf{f}$ RHEED pattern of 2-UC FeSe on ETO. The incident electron beam is along the [100] direction of the substrate for all RHEED images.

\section{RESULTS}

\section{Growth of FeSe/ETO films}

ETO and FeSe layers were prepared in an ultrahigh vacuum (UHV) dual-MBE system. During growth, the sample surface was monitored by reflection high-energy electron diffraction (RHEED). $\left(\mathrm{LaAlO}_{3}\right)_{0.3}\left(\mathrm{Sr}_{2} \mathrm{TaAlO}_{6}\right)_{0.7}(\mathrm{LSAT})$ crystals were used as substrates and the RHEED pattern is shown in Fig. 1a. LSAT has a similar lattice constant $(3.868 \AA)$ to that of ETO $(3.905 \AA)$ and remains insulating after high-temperature growth. ETO films of precisely 20 unit cells (UC) were grown using the conditions as described in ref. ${ }^{48}$ (see also "Methods" for further details). During the growth of $\mathrm{EuTiO}_{3}$ films, the RHEED intensity shows clear oscillations indicating layer-by-layer growth at a rate of $\sim 65$ s per UC (Fig. 1b). The RHEED image of the final ETO film after annealing exhibits sharp spots with the specular spot much stronger than the first-order diffraction spots (Fig. 1c), a signature of a high-quality crystalline film with $\mathrm{TiO}_{2}$-termination layer. This is further evidenced by the atomic force microscope image (Fig. 1d), which shows regular and flat terraces with the step height of $\sim 4 \AA$. FeSe films were grown on ETO using the well-established MBE method $^{49}$.
Typical RHEED images of 1-UC and 2-UC FeSe films are shown in Fig. 1e,f. The film thickness can be simply controlled by the deposition time. The coincident diffraction-streak positions of all the layers indicate similar in-plane lattice constants and lattice orientations, evidence of the high-quality epitaxial FeSe/ETO heterostructure.

\section{X-ray absorption spectroscopy}

X-ray absorption spectroscopy (XAS) and X-ray magnetic circular dichroism (XMCD) were carried out at temperatures down to $2 \mathrm{~K}$ $\left[X-\right.$ Treme beamline, Swiss Light Source $\left.(S L S)^{50}\right]$. XAS and XMCD detected by total electron yield are powerful tools to study the spin polarization with surface sensitivity and elemental selectivity. The geometry of the setup is sketched in Supplementary Fig. 1. The ETO layers in the FeSe/ETO samples show antiferromagnetic ordering. The Eu $\mathrm{M}_{4,5}$-edge spectral shape is consistent with the $\mathrm{Eu}^{2+}$ valence state and exhibits large XMCD in a magnetic field of 6.8 T (Fig. 2a,b). The XMCD intensity, which is proportional to the magnetization $M$, decreases and diminishes with decreasing magnetic field (Fig. 2b) or increasing temperature (Fig. 2c). 

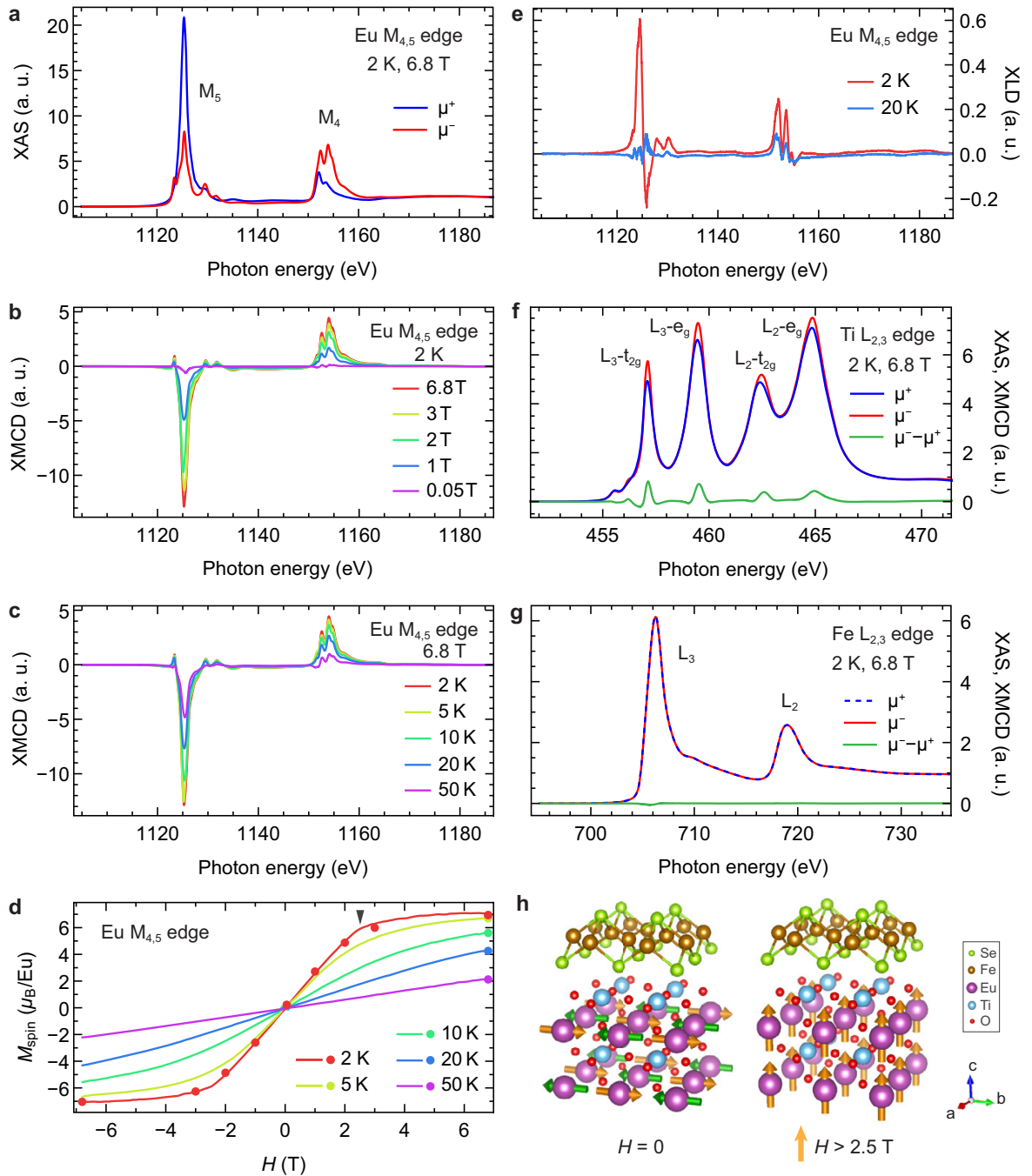

Fig. 2 X-ray absorption spectroscopy (XAS) of FeSe/ETO. a XAS of Eu $\mathrm{M}_{4,5}$ edge at $2 \mathrm{~K}$ in the field of $6.8 \mathrm{~T}$, measured with circularly polarized photons. The incident direction and magnetic field are perpendicular to the sample surface. $\mathbf{b}$ X-ray magnetic circular dichroism (XMCD) at the Eu $\mathrm{M}_{4,5}$ edges at $2 \mathrm{~K}$ in various magnetic fields. $\mathrm{cXMCD}$ at the Eu $\mathrm{M}_{4,5}$ edges in the field of $6.8 \mathrm{~T}$ at various temperatures. $\mathbf{d}$ The magnetization contributed by spins on Eu atoms as a function of magnetic field and temperature. The points are the data extracted from the XMCD spectra using the sum rules. The lines are the XMCD intensities at the Eu $M_{5}$ peak measured with sweeping field, and normalized to the sum-rule values at $6.8 \mathrm{~T}$ for each temperature. e X-ray linear dichroism (XLD) at the Eu $\mathrm{M}_{45}$ edges at zero field at $2 \mathrm{~K}$ and $20 \mathrm{~K}$. The incident direction is $60^{\circ}$ from the surface normal. $\mathbf{f X A S}$ and XMCD at the Ti $L_{2,3}$ edges measured with circularly polarized photons. $\mathbf{g} X A S$ and XMCD at the Fe $L_{2,3}$ edges measured with circularly polarized photons. $\mathbf{h}$ Illustration of the spin alignment in the FeSe/ETO heterostructures with different external fields.

The evolution of $M$ with the applied magnetic field is summarized in Fig. 2d. With no external field, the zero $M$ together with the enhanced X-ray linear dichroism (XLD) at $2 \mathrm{~K}$ (Fig. 2e) reveals the antiferromagnetic ground state of the ETO film.

The $M-H$ curve at $2 \mathrm{~K}$ is saturated above a critical field of $\sim 2.5 \mathrm{~T}$ for $H \| c$ (Fig. 2d), as a result of the complete polarization of the local magnetic moments on Eu atoms. The spin moment at $2 \mathrm{~K}$ and $6.8 \mathrm{~T}$ deduced from the sum rule ${ }^{51}$ is $\sim 6.95 \mu_{\mathrm{B}}$ per Eu atom (see Supplementary Note 1 and Supplementary Fig. 2 for the calculation method), agreeing with seven unpaired electrons in the $4 \mathrm{f}$ shell of each Eu atom ${ }^{46}$. For the field $70^{\circ}$ from the surface normal, the critical field is $\sim 1.2 \mathrm{~T}$ (Supplementary Fig. 3 ), indicating that the easy axis is likely in the $a-b$ plane. This is consistent with neutron-diffraction results showing that the AFM axis is along the (100) direction of the pseudocubic unit cell of ETO ${ }^{52}$. In the presence of an external magnetic field, the local spin moments are reoriented, until fully aligned above the critical field (Fig. 2h). When the temperature exceeds $T_{N}$, the $M-H$ curves become straighter and the magnitude weaker (Fig. 2d) because the spin orientations are randomized by thermal excitations. The behavior in Fig. $2 \mathrm{~d}$ is very similar to that in the direct magnetization measurements of ETO bulk or films in the literature $47,53,54$.

The $\mathrm{Ti}_{2,3}$ edges of ETO are characteristic of the octahedral $\mathrm{Ti}^{4+}$ state (Fig. $2 \mathrm{f}^{55}$. Sizable XMCD is also observed, which is more pronounced than that for STO in FeSe/STO (Supplementary Fig. $4 a$ ), yielding an orbital moment of $-0.19 \pm 0.02 \mu_{\mathrm{B}}$ per Ti (the spin moment is present as well, yet the spin sum rule cannot be applied due to its large error for the Ti L-edge ${ }^{56}$ ). The XMCD of Ti responds to the magnetic field in a similar way to that of Eu with the same critical field (Supplementary Fig. 4b), indicating that spin polarization has been induced on Ti-3d orbitals through exchange interaction with Eu-4f states due to the proximity of the energy bands of these states ${ }^{57}$.

$\mathrm{TiO}_{2}$ layer is the immediate layer between FeSe and EuO layer (Fig. 2h). The $\mathrm{TiO}_{2}-\mathrm{Fe}$ interlayer distance is $\sim 4.3 \AA$ with reference to the $\mathrm{FeSe} / \mathrm{STO}$ case $^{58}$. The EuO-Fe distance is larger by $c_{\text {ETO }} / 2=1.95 \AA$, 

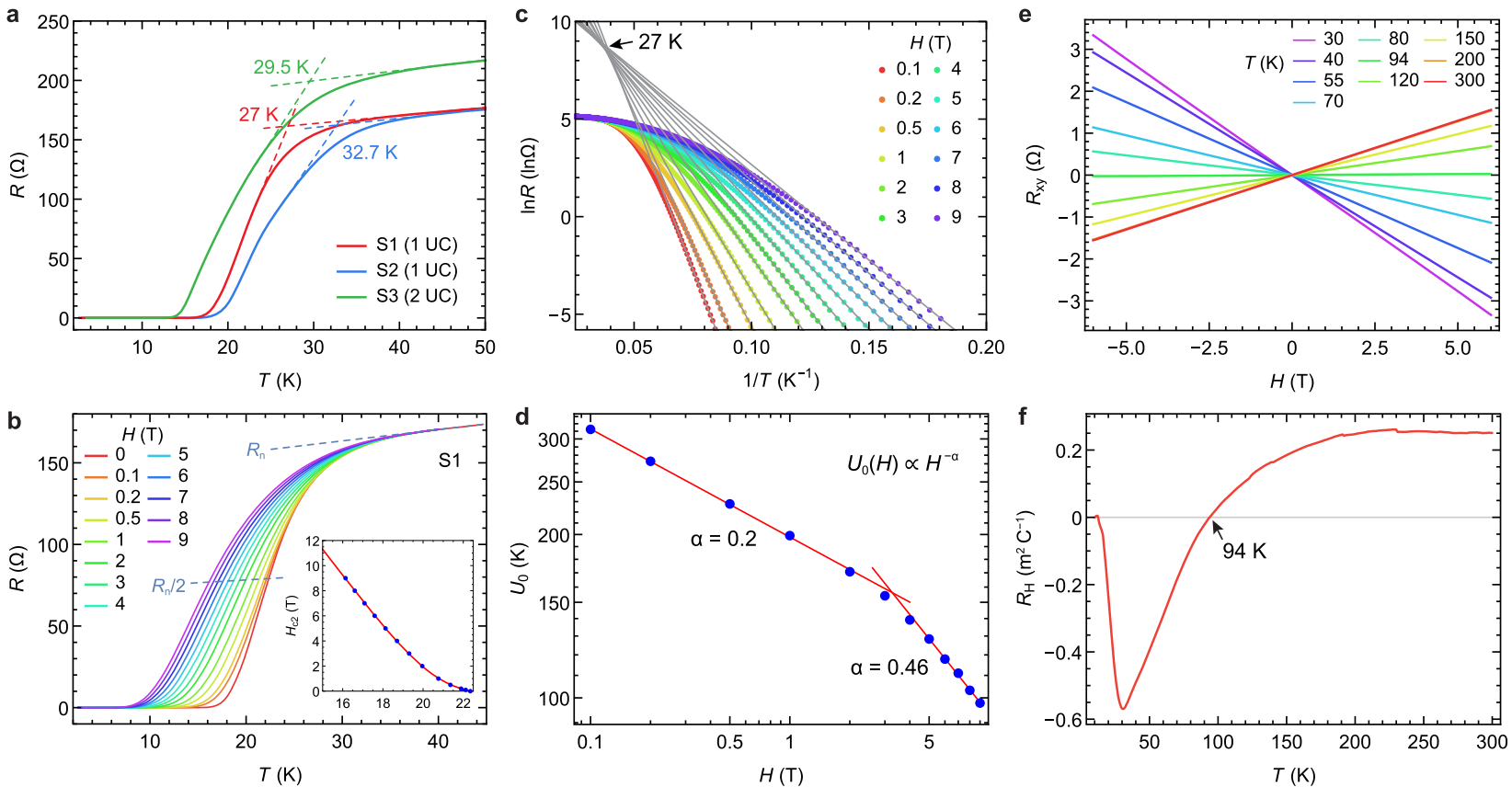

Fig. 3 Transport results of FeSe/ETO. a Temperature dependence of the resistance for three samples with 10-UC FeTe capping layers. The superconducting onset temperatures are marked. $\mathbf{b} R-T$ curves of sample $\mathrm{S} 1$ in various fields with $H \| \mathrm{c}$. The inset shows the upper critical field $H_{\mathrm{c} 2}(T)$ (blue dots) and the fitting with the Ginzburg-Landau model for multigap superconductors (red line). c Arrhenius plot for the resistance in various fields for S1. The corresponding solid lines are fitting results from the Arrhenius relation. d Field dependence of $U_{0}$ (blue dots) derived from the fitting in panel $c$, plotted in log-log scale. The red lines are power-law fittings for the data below and above $3 \mathrm{~T}$, respectively, possessing different exponents. e Hall resistance $R_{\mathrm{xy}}(H)$ of $\mathrm{S} 1$ at various temperatures. $\mathbf{f}$ Temperature dependence of the Hall coefficient obtained as $\left[R_{\mathrm{xy}}(6 \mathrm{~T})-R_{\mathrm{xy}}(-6 \mathrm{~T})\right] / 12 \mathrm{~T}$, showing a sign change at $94 \mathrm{~K}$.

but the Eu magnetic moment is larger than the Ti moment by more than an order of magnitude. A proximity-induced exchange coupling in FeSe from the top EuO and $\mathrm{TiO}_{2}$ layers of ETO should be expected, yet no obvious effect is observed. The XAS at the Fe $L_{2,3}$ edges shows a pure $\mathrm{Fe}^{2+}$ state (Fig. $2 \mathrm{~g}$ ), indicating that the film was well protected from oxidization ${ }^{59}$. The XMCD signal of Fe is negligible $\left(<0.02 \mu_{\mathrm{B}}\right.$ per $\mathrm{Fe}$ ) at the maximum field of $6.8 \mathrm{~T}$ that we could apply, which suggests small magnetic susceptibility or no static spin order in FeSe, although local spin fluctuations cannot be excluded.

\section{Magnetotransport measurements}

The superconductivity of the FeSe/ETO heterostructure is characterized by electrical and magneto- transport measurements in Fig. 3. The ETO films are insulating with sheet resistance higher than $4 \times 10^{5} \Omega \cdot \mathrm{sq}^{-1}$ at room temperature ${ }^{48}$, indicating a low doping level consistent with the $\mathrm{Eu}^{2+}$ and $\mathrm{Ti}^{4+}$ states. Hence, the conductivity of the FeSe plus FeTe cap dominates the transport measurements. One of our most important findings is that monolayer FeSe on ETO hosts hightemperature superconductivity with the onset at around $30 \mathrm{~K}$ as shown in Fig. 3a, which has been reproduced in multiple samples and is comparable to our FeSe/STO samples prepared in the similar conditions ${ }^{49}$. No systematic difference between the $T_{\mathrm{c}}$ of 1-UC and 2-UC FeSe films is observed, supporting the conclusion of interfacial superconductivity. The $R-T$ curve displays a two-step transition in some samples, which could be interpreted as the sequential formation of local superconducting puddles and phase-fluctuation states before entering the $2 \mathrm{D}$ superconductivity, as is observed in $\mathrm{LaAlO}_{3} / \mathrm{SrTiO}_{3}{ }^{60}$ and monolayer $\mathrm{Bi}_{2} \mathrm{Sr}_{2} \mathrm{CaCu}_{2} \mathrm{O}_{8+\delta}{ }^{61}$. Figure $3 \mathrm{~b}$ shows the suppression of the transition by an external field along the $c$ axis. The upper critical field $H_{\mathrm{c} 2}(T)$ is extracted adopting the midpoint criterion: $R\left(H_{\mathrm{c} 2}, T\right)=0.5 R_{\mathrm{n}}(T)$, where $R_{\mathrm{n}}(T)$ is the normal stated resistance extrapolated from above the
$T_{c}$ onset. The $T_{c}$ shows a faster suppression in low fields and enters a linear region at high fields (inset of Fig. $3 \mathrm{~b}$ ). This can be fit with the Ginzburg-Landau multigap model ${ }^{62}$, yielding $H_{\mathrm{c} 2}(0)$ $=43 \mathrm{~T}$ and therefore the coherence length $\xi=\sqrt{\Phi_{0} / 2 \pi H_{c 2}(0)}$ $=2.8 \mathrm{~nm}\left(\Phi_{0}\right.$ is the magnetic flux quantum), close to that of FeSe/STO $(2.4 \mathrm{~nm})^{17}$ and much larger than the thickness of single-layer FeSe, which confirms the 2D nature of the superconductivity.

As a type-II superconductor with a high upper critical field, FeSe exhibits rich vortex phenomena in the mixed state. Thermal fluctuations in FeSe films can result in thermally activated flux flow (TAFF) behavior where the vortices hop between neighboring pinning centers ${ }^{63,64}$. In the TAFF region near $T_{c}$, the resistance and temperature can be described by the Arrhenius relation $R(T, H)=R_{0}(H) \exp \left[-U_{0}(H) / T\right]$ or $\ln R(T, H)=$ In $R_{0}(H)-U_{0}(H) / T$, where $R_{0}(H)$ is a temperature-independent constant and $U_{0}(H)$ is the activation energy of flux flow. As shown in Fig. 3c, the experimental data are well fit by this relation. The fitting lines for different magnetic fields extrapolate to the same point corresponding to a temperature of $27 \mathrm{~K}$, in agreement with $T_{\mathrm{c}}{ }^{\text {onset }}$. Furthermore, the activation energy $U_{0}$ is obtained as the slope of the lines, and follows the power-law relation with the field, $U_{0}(H) \propto H^{-a}$. As shown in Fig. $3 \mathrm{~d}, a$ is field dependent with $a=0.20$ for $H<3 \mathrm{~T}$ and $a=0.46$ for $H>3 \mathrm{~T}$, implying the crossover from the single-vortex pinning at low field to the collective pinning at high field, which is a typical behavior in Fe chalcogenides ${ }^{64}$.

Similar to Hall data from previous reports on FeTe-capped monolayer $\mathrm{FeSe} / \mathrm{STO}^{65}$, the Hall coefficient changes sign from positive to negative as temperature is decreased (Fig. 3e,f). At low temperatures where superconductivity emerges, the dominant carriers are electrons, hinting at increased electron doping in the FeSe layer caused by charge transfer from ETO. 


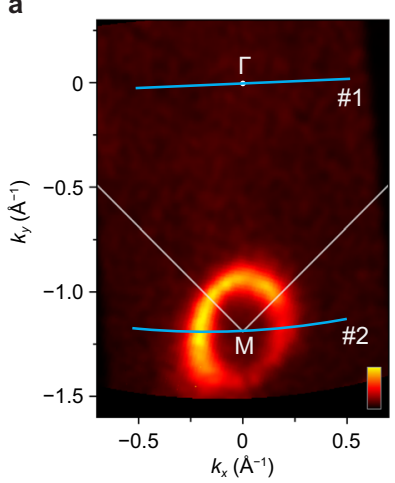

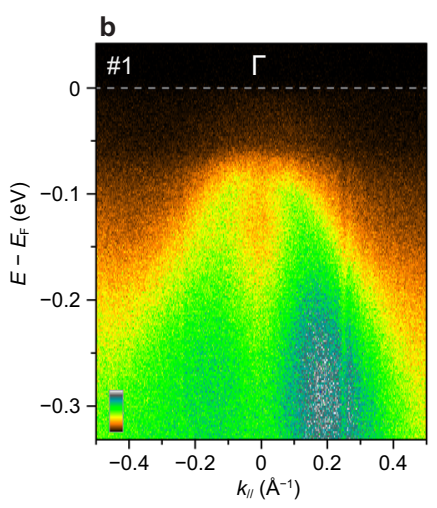

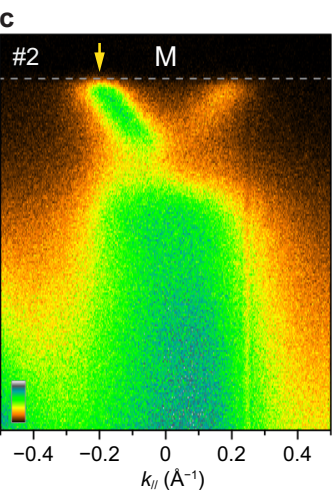

Fig. 4 Angle-resolved photoemission spectroscopy (ARPES) results of 1-UC FeSe/ETO. a Constant energy map at the Fermi energy covering $\Gamma$ and one of the $M$ points of the Brillouin zone. The gray line indicates the first Brillouin zone boundary. $\mathbf{b}$ and $\mathbf{c}$ Photoemission-intensity images along cut \#1 and cut \#2 in panel a, respectively. $\mathbf{d}$ Symmetrized energy distribution curve (EDC) taken at the Fermi momentum near M point, marked by the arrow in panel $c$. The blue line is a fit based on the BCS self-energy function with a gap size of 12 meV.

\section{Angle-resolved photoemission spectroscopy}

The doping in FeSe is directly evidenced in the ARPES measurements at $10 \mathrm{~K}$, indicating charge transfer from ETO to FeSe. Although the concentration of oxygen vacancies in ETO might be different from STO, recent studies have shown that the charge transfer is caused by the band alignment near the interface and that the resulting doping in FeSe is insensitive to the carrier density in the neighboring oxide layers ${ }^{26}$. As shown in Fig. $4 a$, the 1-UC FeSe on ETO has a large elliptical Fermi surface at the $M$ point of the Brillouin zone with no Fermi surface at the $\Gamma$ point. One can see from the dispersion images that the hole-like band at the $\Gamma$ point is $70 \mathrm{meV}$ below the Fermi level (Fig. 4b), hence, only the electron-like band at the $M$ point (Fig. 4c) contributes the carriers and the Cooper pairs. The doping level estimated from Fermi surface size based on the Luttinger's theorem is $\sim 0.11$ electrons per Fe. We observe an energy gap $\Delta=12 \mathrm{meV}$ at the Fermi level on the electron band (Fig. 4d), consistent with the high- $T_{c}$ superconductivity from transport. The fermiology, band structure, and superconducting gap of 1-UC FeSe/ETO closely resemble those of the 1-UC FeSe/STO ${ }^{24}$, so they should have a common mechanism for their enhancement of superconductivity.

\section{DISCUSSION}

We have grown monolayer FeSe epitaxially on an antiferromagnetic ETO layer. In spite of its proximity, no evidence of magnetic order in FeSe is seen in the XMCD measurements. This implies that monolayer FeSe is robust against forming a static magnetic order. Nevertheless, at the interface where antiferromagnetic order diminishes, the spin fluctuation is likely to be strong, which might play a role in the Cooper pairing.

As proposed by some theoretical studies, the Cooper pairing in monolayer FeSe is mediated by AFM spin fluctuations, and $T_{C}$ should be enhanced when FeSe is stacked with a titanate that can provide charge transfer and electron-phonon coupling or an AFM material that can induce stronger spin fluctuations ${ }^{31}$. Intriguingly, ETO fulfills both demands at the same time, and the high- $T_{c}$ superconductivity is indeed realized in the monolayer FeSe/ETO, adding another member to the FeSe-oxide interface family.

No further enhancement in $T_{c}$ compared with FeSe/STO was observed, likely because the $T_{\mathrm{N}}$ of ETO is lower than the $T_{\mathrm{c}}$ of FeSe film, or the interface exchange interaction is not crucial to boost the superconductivity in this system. It was reported that in contrast to FeSe/STO, high- $T_{\mathrm{c}}$ superconductivity is absent in FeS/ $\mathrm{STO}^{66}$, even though they possess similar electron doping and spin dynamics. Recent theoretical work points out that the phonons in the surface layer of $\mathrm{TiO}_{2}$ are responsible for pairing, which is dependent on the $\mathrm{FeSe}-\mathrm{TiO}_{2}$ distance ${ }^{40}$. In this framework, the spin fluctuation does not dominate the superconductivity of FeSe/ titanate. If the $\mathrm{FeSe}-\mathrm{TiO}_{2}$ distance is similar in $\mathrm{FeSe} / \mathrm{ETO}$ and $\mathrm{FeSe} /$ STO, then a similar $T_{c}$ should be expected. Besides ETO, $\mathrm{LaTiO}_{3}$ is also found to boost the superconductivity of $\mathrm{FeSe}^{26}$, adding more support to this scenario. Further theoretical study of the FeSe/ETO interface is warranted.

We are also able to switch ETO from antiferromagnetic to ferromagnetic ordering by an applied external magnetic field. Importantly, the field required to switch ETO (2.5 T) is well below the $H_{\mathrm{c} 2}$ of the FeSe on top, indicating that the superconductivity persists during the magnetic moment realignment. The tunable spin polarization, high- $T_{\mathrm{c}}$ and high- $H_{\mathrm{c} 2}$ superconductivity and the nanometer-scale thickness makes the FeSe/ETO heterostructure appropriate for potential applications in functional devices.

Furthermore, when epitaxial FeTe, which is a bicollinear antiferromagnet with $2.25 \mu_{\mathrm{B}}$ per Fe and $T_{\mathrm{N}} \sim 70 \mathrm{~K}^{67,68}$, is used as the capping layer, the FeTe/FeSe/ETO heterostructure forms an AFM-SC-AFM sandwich that is an interesting platform supporting exotic superconducting properties in the presence of diverse exchange interactions. Our work makes an important step toward the design of functional interfaces, specifically the precise construction of oxide/chalcogenide interfaces. Further exploration would be aimed at searching for higher Néel temperature oxide materials, so that the spin interaction would be stronger.

In conclusion, we successfully grew heterostructures of monolayer FeSe on $\mathrm{EuTiO}_{3}$. The ETO layer is AFM-FM switchable with external field, while the FeSe layer is superconducting at temperatures up to $30 \mathrm{~K}$. The integration of magnetic order and superconductivity in this system will trigger the study of the interaction between charge and spin, and promote the development of superconductors and spintronics in the form of artificial heterostructures.

\section{METHODS}

\section{Sample preparation}

The films were grown in a Veeco GENXplor dual-MBE system with a base pressure lower than $5 \times 10^{-10}$ Torr. High-purity $\mathrm{Eu}, \mathrm{Ti}, \mathrm{Fe}$, and $\mathrm{Te}$ was evaporated from separated Knudsen cells. Se was evaporated from a Se cracker. The source fluxes were measured by a quartz-crystal microbalance and further calibrated by RHEED oscillations during growth. The oxygengas flux was controlled by a leak valve and the partial pressure was monitored by a residual gas analyzer. The electron beam energy of RHEED was $10 \mathrm{keV}$. LSAT(001) single-crystal substrates (CrysTec $\mathrm{GmbH}$ ) were degassed at $700{ }^{\circ} \mathrm{C}$ for $30 \mathrm{~min}$ in the oxide MBE chamber before growth. $\mathrm{EuTiO}_{3}$ films were grown by codepositing $\mathrm{Eu}$ and $\mathrm{Ti}$ with calibrated flux ratio on the substrates at $700{ }^{\circ} \mathrm{C}$ with the $\mathrm{O}_{2}$ partial pressure at $4 \times 10^{-8}$ Torr, followed by annealing at $700^{\circ} \mathrm{C}$ for $0.5 \mathrm{~h}$. The samples were then transferred through a UHV transfer line to the chalcogenide MBE chamber 
for FeSe growth. The flux ratio between Se and Fe was $\sim 5$. The substrate temperature was $420^{\circ} \mathrm{C}$ for growth and $480^{\circ} \mathrm{C}$ for annealing. Extra Fe was deposited to compensate Fe vacancies in the film ${ }^{49}$. The samples for transport measurements were capped with $10-\mathrm{UC}$ FeTe at $280^{\circ} \mathrm{C}$ and those for XAS and ARPES measurements were capped with $15 \mathrm{~nm}$ Te and $5 \mathrm{~nm}$ Se successively at room temperature before being exposed to the air.

\section{XAS measurements}

XAS measurements were performed at the EPFL-PSI X-Treme beamline of the Swiss Light Source ${ }^{50}$. The capped FeSe/ETO samples were sealed in vacuum sealer bags, operating inside an Ar-filled glove box at UBC. The samples were shipped to SLS in two days. Then they were mounted on the sample holders in the air in $10 \mathrm{~min}$ and then loaded into the chamber. The films were decapped by annealing at $300^{\circ} \mathrm{C}$ in a UHV preparation chamber and then transferred to the measurement chamber where the base pressure was $5 \times 10^{-11}$ Torr. The base temperature of the cryostat was 2.0 K. The sample surface was placed in the vertical plane. The X-ray beam and magnetic field are horizontal and parallel to each other. Total electron yield was collected as the XAS signal. The XMCD signal was obtained as the difference between the XAS spectra acquired with the photon helicity vector parallel $\left(\mu^{-}\right)$and antiparallel $\left(\mu^{+}\right)$to the magnetic field. The XLD signal was obtained as the difference between the XAS spectra acquired with the linearly horizontal (LH) and linearly vertical (LV) polarizations at an incidence angle of $60^{\circ}$ from the surface normal. The LV-polarization vector was parallel to the surface and $\mathrm{LH}$ was $60^{\circ}$ from the surface plane. For each $\mathrm{XMCD}$ or $\mathrm{XLD}$, multiple XAS curves were acquired in a sequence alternating the polarization at every spectrum and were averaged afterward for each polarization. The magnetic moments were calculated using the sum rule as described in Supplementary Information.

\section{Transport measurements}

Electrical transport properties were measured by the four-terminal method in a Quantum Design physical property measurement system (PPMS). The magnetic field was perpendicular to the sample surface. Gold wires were cold-bonded on the samples with indium lumps as the electrodes.

\section{ARPES}

ARPES measurements were carried out at UBC on a lab-based system. The capped samples were transferred from the MBE and mounted on the copper-sample holders for ARPES, being exposed to the air for $5 \mathrm{~min}$. Conductive carbon paste was placed on the edges of the films for grounding. The samples were decapped in the preparation chamber at $420^{\circ} \mathrm{C}$ for $2 \mathrm{~h}$ before measurements. The ARPES chamber is equipped with a Scienta VUV5k helium-discharge lamp and a Scienta DA30-L electron analyzer. The photon energy is $21.2 \mathrm{eV}$. Energy and angle resolutions are better than $8 \mathrm{meV}$ and 0.4 degrees. The temperature of the sample was kept below $10 \mathrm{~K}$ during the measurements. The pressure of the chamber was below $6 \times 10^{-11}$ Torr. The superconducting gap is determined by fitting the symmetrized EDC to a spectral function with the simplified BCS self-energy ${ }^{69}, \Sigma(\mathbf{k}, \omega)=-\mathrm{i} \Gamma_{1} \Delta^{2} /\left[\omega+\varepsilon(\mathbf{k})+\mathrm{i} \Gamma_{0}\right]$, where $\omega$ is the energy relative to Fermi energy, $\Delta$ is the gap size, $\Gamma_{0}$ is the pair-breaking scattering rate, $\Gamma_{1}$ represents the single-particle scattering rate, and $\varepsilon(\mathbf{k})$ is the band dispersion $\left[\varepsilon\left(\mathbf{k}_{\mathbf{F}}\right)=0\right]$.

\section{DATA AVAILABILITY}

The data that support the findings of this study are available from the corresponding authors upon request.

Received: 3 July 2021; Accepted: 16 September 2021; Published online: 07 October 2021

\section{REFERENCES}

1. Wolf, S. A. et al. Spintronics: a spin-based electronics vision for the future. Science 294, 1488-1495 (2001).

2. De Gennes, P. G. Coupling between ferromagnets through a superconducting layer. Phys. Lett. 23, 10-11 (1966).

3. Linder, J. \& Robinson, J. W. A. Superconducting spintronics. Nat. Phys. 11, 307-315 (2015).
4. Tao, Y. C. \& Hu, J. G. Superconducting spintronics: spin-polarized transport in superconducting junctions with ferromagnetic semiconducting contact. J. Appl. Phys. 107, 041101 (2010).

5. Ohtomo, A. \& Hwang, H. Y. A high-mobility electron gas at the $\mathrm{LaAlO}_{3} / \mathrm{SrTiO}_{3}$ heterointerface. Nature 427, 423-426 (2004).

6. Bert, J. A. et al. Direct imaging of the coexistence of ferromagnetism and superconductivity at the $\mathrm{LaAlO}_{3} / \mathrm{SrTiO}_{3}$ interface. Nat. Phys. 7, 767-771 (2011).

7. Dikin, D. A. et al. Coexistence of superconductivity and ferromagnetism in two dimensions. Phys. Rev. Lett. 107, 056802 (2011).

8. Li, L., Richter, C., Mannhart, J. \& Ashoori, R. C. Coexistence of magnetic order and two-dimensional superconductivity at $\mathrm{LaAlO}_{3} / \mathrm{SrTiO}_{3}$ interfaces. Nat. Phys. 7, 762-766 (2011).

9. Stornaiuolo, D. et al. Tunable spin polarization and superconductivity in engineered oxide interfaces. Nat. Mater. 15, 278-283 (2015).

10. Wittlich, P. et al. Exploring possible ferromagnetism of the $\mathrm{LaAlO}_{3} / \mathrm{SrTiO}_{3}$ interface. Phys. Rev. Mater. 3, 104418 (2019).

11. Hsu, F.-C. et al. Superconductivity in the PbO-type structure a-FeSe. Proc. Natl. Acad. Sci. USA 105, 14262-14264 (2008).

12. Watson, M. D. et al. Emergence of the nematic electronic state in FeSe. Phys. Rev. B 91, 115106 (2015).

13. Bohmer, A. E. \& Kreisel, A. Nematicity, magnetism and superconductivity in FeSe. J. Phys. Condens. Matter 30, 023001 (2018).

14. Baek, S. H. et al. Orbital-driven nematicity in FeSe. Nat. Mater. 14, 210-214 (2015).

15. Wang, Q. et al. Magnetic ground state of FeSe. Nat. Commun. 7, 12182 (2016).

16. Wang, Q.-Y. et al. Interface-induced high-temperature superconductivity in single unit-cell FeSe films on $\mathrm{SrTiO}_{3}$. Chin. Phys. Lett. 29, 037402 (2012).

17. Zhou, G. et al. Interface induced high temperature superconductivity in single unit-cell FeSe on $\mathrm{SrTiO}_{3}$ (110). Appl. Phys. Lett. 108, 202603 (2016).

18. Pedersen, A. K. et al. Interfacial superconductivity in FeSe ultrathin films on $\mathrm{SrTiO}_{3}$ probed by in situ independently driven four-point-probe measurements. Phys. Rev. Lett. 124, 227002 (2020).

19. $\mathrm{He}, \mathrm{S}$. et al. Phase diagram and electronic indication of high-temperature superconductivity at $65 \mathrm{~K}$ in single-layer FeSe films. Nat. Mater. 12, 605-610 (2013).

20. Zhang, Z. et al. Onset of the Meissner effect at $65 \mathrm{~K}$ in FeSe thin film grown on Nb-doped $\mathrm{SrTiO}_{3}$ substrate. Sci. Bull. 60, 1301-1304 (2015).

21. Zhou, G. et al. Interface enhanced superconductivity in monolayer FeSe films on $\mathrm{MgO}(001)$ : charge transfer with atomic substitution. Sci. Bull. 63, 747-752 (2018).

22. Yang, $\mathrm{H}$. et al. Superconductivity above $28 \mathrm{~K}$ in single unit cell FeSe films interfaced with $\mathrm{GaO}_{2-\delta}$ layer on $\mathrm{NdGaO}_{3}(110)$. Sci. Bull. 64, 490-494 (2019).

23. Rebec, S. N. et al. Coexistence of replica bands and superconductivity in FeSe monolayer films. Phys. Rev. Lett. 118, 067002 (2017).

24. Liu, D. et al. Electronic origin of high-temperature superconductivity in singlelayer FeSe superconductor. Nat. Commun. 3, 931 (2012).

25. Tan, S. et al. Interface-induced superconductivity and strain-dependent spin density waves in $\mathrm{FeSe} / \mathrm{SrTiO}_{3}$ thin films. Nat. Mater. 12, 634-640 (2013).

26. Jia, T. et al. Magic doping and robust superconductivity in monolayer FeSe on titanates. Adv. Sci. 8, 2003454 (2021).

27. Peng, R. et al. Tuning the band structure and superconductivity in single-layer FeSe by interface engineering. Nat. Commun. 5, 5044 (2014).

28. Huang, D. \& Hoffman, J. E. Monolayer FeSe on $\mathrm{SrTiO}_{3}$. Annu. Rev. Condens. Matter Phys. 8, 311-336 (2017).

29. Agterberg, D., Shishidou, T., O'Halloran, J., Brydon, P. \& Weinert, M. Resilient nodeless d-wave superconductivity in monolayer FeSe. Phys. Rev. Lett. 119, 267001 (2017).

30. Gao, Y., Yu, Y., Zhou, T., Huang, H. \& Wang, Q.-H. Possible spin excitation structure in monolayer FeSe grown on $\mathrm{SrTiO}_{3}$. Phys. Rev. B 96, 014515 (2017).

31. Lee, D.-H. Routes to high-temperature superconductivity: a lesson from FeSe/ $\mathrm{SrTiO}_{3}$. Annu. Rev. Condens. Matter Phys. 9, 261-282 (2018).

32. Miyata, Y., Nakayama, K., Sugawara, K., Sato, T. \& Takahashi, T. High-temperature superconductivity in potassium-coated multilayer FeSe thin films. Nat. Mater. 14, 775-779 (2015).

33. Tang, C. et al. Interface-enhanced electron-phonon coupling and hightemperature superconductivity in potassium-coated ultrathin FeSe films on $\mathrm{SrTiO}_{3}$. Phys. Rev. B 93, 020507 (2016).

34. Zhang, $H$. et al. Origin of charge transfer and enhanced electron-phonon coupling in single unit-cell FeSe films on $\mathrm{SrTiO}_{3}$. Nat. Commun. 8, 214 (2017).

35. Shiogai, J., Ito, Y., Mitsuhashi, T., Nojima, T. \& Tsukazaki, A. Electric-field-induced superconductivity in electrochemically etched ultrathin FeSe films on $\mathrm{SrTiO}_{3}$ and MgO. Nat. Phys. 12, 42-46 (2015).

36. Zhang, S. et al. Lattice dynamics of ultrathin FeSe films on $\mathrm{SrTiO}_{3}$. Phys. Rev. B 97, 035408 (2018).

37. Lee, J. J. et al. Interfacial mode coupling as the origin of the enhancement of $T_{c}$ in FeSe films on $\mathrm{SrTiO}_{3}$. Nature 515, 245-248 (2014). 
38. Zhang, $C$. et al. Ubiquitous strong electron-phonon coupling at the interface of $\mathrm{FeSe} / \mathrm{SrTiO}_{3}$. Nat. Commun. 8, 14468 (2017).

39. Song, Q. et al. Evidence of cooperative effect on the enhanced superconducting transition temperature at the $\mathrm{FeSe} / \mathrm{SrTiO}_{3}$ interface. Nat. Commun. 10, 758 (2019).

40. Rosenstein, B. \& Shapiro, B. Y. Phonon mechanism explanation of the superconductivity dichotomy between $\mathrm{FeSe}$ and $\mathrm{FeS}$ monolayers on $\mathrm{SrTiO}_{3}$ and other substrates. Phys. Rev. B 103, 224517 (2021).

41. Liu, C. et al. High-order replica bands in monolayer $\mathrm{FeSe} / \mathrm{SrTiO}_{3}$ revealed by polarization-dependent photoemission spectroscopy. Nat. Commun. 12, 4573 (2021).

42. Zheng, F., Wang, Z., Kang, W. \& Zhang, P. Antiferromagnetic FeSe monolayer on $\mathrm{SrTiO}_{3}$ : the charge doping and electric field effects. Sci. Rep. 3, 2213 (2013).

43. Wang, Z. F. et al. Topological edge states in a high-temperature superconductor $\mathrm{FeSe} / \mathrm{SrTiO}_{3}(001)$ film. Nat. Mater. 15, 968-973 (2016).

44. Zhou, Y. et al. Antiferromagnetic order in epitaxial FeSe films on $\mathrm{SrTiO}_{3}$. Phys. Rev. Lett. 120, 097001 (2018).

45. Pelliciari, J. et al. Evolution of spin excitations from bulk to monolayer FeSe. Nat. Commun. 12, 3122 (2021).

46. McGuire, T. R., Shafer, M. W., Joenk, R. J., Alperin, H. A. \& Pickart, S. J. Magnetic structure of $\mathrm{EuTiO}_{3}$. J. Appl. Phys. 37, 981-982 (1966).

47. Midya, A. et al. Large adiabatic temperature and magnetic entropy changes in $\mathrm{EuTiO}_{3}$. Phys. Rev. B 93, 094422 (2016).

48. Shin, H. et al. Controlling the electrical and magnetic ground states by doping in the complete phase diagram of titanate $\mathrm{Eu}_{1-\mathrm{x}} \mathrm{La}_{\mathbf{x}} \mathrm{TiO}_{3}$ thin films. Phys. Rev. B 101, 214105 (2020).

49. Liu, C. \& Zou, K. Tuning stoichiometry and its impact on superconductivity of monolayer and multilayer $\mathrm{FeSe}$ on $\mathrm{SrTiO}_{3}$. Phys. Rev. B 101, 140502(R) (2020).

50. Piamonteze, C. et al. X-Treme beamline at SLS: X-ray magnetic circular and linear dichroism at high field and low temperature. J. Synchrotron Radiat. 19, 661-674 (2012).

51. Carra, P., Thole, B. T., Altarelli, M. \& Wang, X. X-ray circular dichroism and local magnetic fields. Phys. Rev. Lett. 70, 694-697 (1993).

52. Scagnoli, V. et al. EuTiO 3 magnetic structure studied by neutron powder diffraction and resonant X-ray scattering. Phys. Rev. B 86, 094432 (2012).

53. Takahashi, K. S., Onoda, M., Kawasaki, M., Nagaosa, N. \& Tokura, Y. Control of the anomalous hall effect by doping in $\mathrm{Eu}_{1-\mathrm{x}} \mathrm{La}_{\mathbf{x}} \mathrm{TiO}_{3}$ thin films. Phys. Rev. Lett. 103 057204 (2009).

54. Takahashi, K. S. et al. Anomalous Hall effect derived from multiple Weyl nodes in high-mobility $\mathrm{EuTiO}_{3}$ films. Sci. Adv. 4, eaar7880 (2018).

55. Krüger, $\mathrm{P}$. Multichannel multiple scattering calculation of $\mathrm{L}_{2,3}$-edge spectra of $\mathrm{TiO}_{2}$ and $\mathrm{SrTiO}_{3}$ : Importance of multiplet coupling and band structure. Phys. Rev. B 81, 125121 (2010).

56. Piamonteze, C., Miedema, P. \& de Groot, F. M. F. Accuracy of the spin sum rule in $\mathrm{XMCD}$ for the transition-metalLedges from manganese to copper. Phys. Rev. B 80, 184410 (2009).

57. Maruhashi, K. et al. Anisotropic quantum transport through a single spin channel in the magnetic semiconductor $\mathrm{EuTiO}_{3}$. Adv. Mater. 32, 1908315 (2020).

58. Peng, R. et al. Picoscale structural insight into superconductivity of monolayer $\mathrm{FeSe} / \mathrm{SrTiO}_{3}$. Sci. Adv. 6, eaay4517 (2020).

59. Chen, C. L. et al. X-Ray spectra and electronic correlations of FeSe $\mathrm{Fin}_{\mathrm{x}} \mathrm{Te}_{\mathrm{x}}$. Phys. Chem. Chem. Phys. 13, 15666-15672 (2011).

60. Chen, Z. et al. Carrier density and disorder tuned superconductor-metal transition in a two-dimensional electron system. Nat. Commun. 9, 4008 (2018).

61. $\mathrm{Yu}, \mathrm{Y}$. et al. High-temperature superconductivity in monolayer $\mathrm{Bi}_{2} \mathrm{Sr}_{2} \mathrm{CaCu}_{2} \mathrm{O}_{8+\delta}$ Nature 575, 156-163 (2019).

62. Zhitomirsky, M. E. \& Dao, V. H. Ginzburg-Landau theory of vortices in a multigap superconductor. Phys. Rev. B 69, 054508 (2004).

63. Sun, Y. et al. High temperature superconducting FeSe films on $\mathrm{SrTiO}_{3}$ substrates. Sci. Rep. 4, 6040 (2014).

64. Lei, H. et al. Iron chalcogenide superconductors at high magnetic fields. Sci. Technol. Adv. Mater. 13, 054305 (2012).

65. Zhang, W. et al. Interface charge doping effects on superconductivity of singleunit-cell FeSe films on $\mathrm{SrTiO}_{3}$ substrates. Phys. Rev. B 89, 060506(R) (2014).

66. Shigekawa, K. et al. Dichotomy of superconductivity between monolayer FeS and FeSe. Proc. Natl. Acad. Sci. USA 116, 24470-24474 (2019).
67. Bao, W. et al. Tunable $(\delta \pi, \delta \pi)$-type antiferromagnetic order in $\mathrm{a}-\mathrm{Fe}(\mathrm{Te}, \mathrm{Se})$ superconductors. Phys. Rev. Lett. 102, 247001 (2009).

68. $\mathrm{Li}, \mathrm{S}$. et al. First-order magnetic and structural phase transitions in $\mathrm{Fe}_{1+\mathrm{y}} \mathrm{Se}_{\mathrm{x}} \mathrm{Te}_{1-\mathrm{x}}$ Phys. Rev. B 79, 054503 (2009).

69. Norman, M. R., Randeria, M., Ding, H. \& Campuzano, J. C. Phenomenology of the low-energy spectral function in high- $\mathrm{T}_{\mathrm{c}}$ superconductors. Phys. Rev. B 57, R11093-R11096 (1998)

\section{ACKNOWLEDGEMENTS}

We are grateful for helpful discussions with Bernhard Keimer, Hidenori Takagi, and George Sawatzky. This research was undertaken, thanks in part to funding from the Max Planck-UBC-UTokyo Centre for Quantum Materials and the Canada First Research Excellence Fund, Quantum Materials and Future Technologies Program. The work at UBC was also supported by the Natural Sciences and Engineering Research Council of Canada (NSERC) and the Canada Foundation for Innovation (CFI). A. Damascelli also acknowledges the support from the British Columbia Knowledge Development Fund (BCKDF), the Gordon and Betty Moore Foundation's EPiQS Initiative, Grant No. GBMF4779, the Canada Research Chairs Program, and the CIFAR Quantum Materials Program. The work at SLS was supported by the PSI-CROSS initiative.

\section{AUTHOR CONTRIBUTIONS}

C.L., H.S. and K.Z. conceived and designed the experiments. H.S. grew the ETO films C.L. grew the FeSe films. C.L., H.S., A. Doll and B.A.D. carried out the XAS experiments. A. Doll, J.D. and C.P. maintained the X-Treme beamline and endstation. C.L. carried out the transport measurements. C.L., H.S., H.-H.K., R.P.D., G.L. and A. Damascelli carried out the ARPES measurements. C.L. and H.S. analyzed the data. C.L. and K.Z. wrote the paper. All authors discussed the results and commented on the paper. C.L. and H.S. contributed equally to this work.

\section{COMPETING INTERESTS}

The authors declare no competing interests.

\section{ADDITIONAL INFORMATION}

Supplementary information The online version contains supplementary material available at https://doi.org/10.1038/s41535-021-00388-5.

Correspondence and requests for materials should be addressed to Chong Liu or Ke Zou.

Reprints and permission information is available at http://www.nature.com/ reprints

Publisher's note Springer Nature remains neutral with regard to jurisdictional claims in published maps and institutional affiliations.

Open Access This article is licensed under a Creative Commons Attribution 4.0 International License, which permits use, sharing, adaptation, distribution and reproduction in any medium or format, as long as you give appropriate credit to the original author(s) and the source, provide a link to the Creative Commons license, and indicate if changes were made. The images or other third party material in this article are included in the article's Creative Commons license, unless indicated otherwise in a credit line to the material. If material is not included in the article's Creative Commons license and your intended use is not permitted by statutory regulation or exceeds the permitted use, you will need to obtain permission directly from the copyright holder. To view a copy of this license, visit http://creativecommons. org/licenses/by/4.0/.

(C) The Author(s) 2021 